\title{
Validação de três folhetos informativos sobre diabetes, sua terapêutica e exercício físico
}

Inês Rosendo, ${ }^{1}$ Luiz Miguel Santiago ${ }^{2}$

\section{RESUMO}

Objetivos: Validação de três folhetos para pessoas com diabetes.

Tipo de estudo: Validação de folhetos.

Local: Internet e USF Rio Dão.

População: Peritos e pessoas com diabetes tipo 2.

Métodos: Desenvolvimento de conteúdos segundo as recomendações existentes, revisão por peritos científicos e de língua e avaliação por pessoas com diabetes tipo 2, avaliação posterior por aplicação de fórmula de inteligibilidade de Flesch adaptada para a língua portuguesa.

Resultados: Na fase de revisão de folhetos receberam-se 14 contribuições de peritos científicos, uma de pessoa com diabetes e duas de peritos de língua. Realizaram-se todas as alterações propostas e retiraram-se as frases que suscitavam dúvidas ou opiniões contrárias. Na fase de avaliação dos folhetos foram recebidas colaborações de 10 pessoas com diabetes, $50 \%$ do género masculino, com idade média de $63,0 \pm 10,1$ anos e formação média de 5,6 $\pm 4,5$ anos. A avaliação da inteligibilidade dos folhetos revelou um grau de legibilidade dos mesmos ao nível do $9^{\circ}-12^{\circ}$ ano. O número de palavras e sílabas totais aumentaram em todos os folhetos, desde a fase inicial até à final.

Conclusões: Este estudo foi inovador no sentido de validar folhetos para informação a pessoas com diabetes em Portugal, usando simultaneamente validação por peritos científicos e de língua (que fizeram grandes alterações ao folheto), envolvendo os pacientes na validação (que revelaram poucas dúvidas) e fazendo uma avaliação de inteligibilidade final (que revelou um nível provavelmente demasiado exigente). Sugere-se o desenvolvimento de ferramentas realizadas no contexto Português para avaliação de inteligibilidade, qualidade de conteúdos, adaptação à literacia em saúde dos pacientes-alvo e, especialmente, do nível de compreensão e melhoria da capacitação dos mesmos.

Palavras-chave: Folhetos; Prospeto para educação de pacientes; Diabetes mellitus; Terapêutica; Exercício

\section{INTRODUÇÃO}

1 diabetes, sendo um problema de saúde pública de elevada magnitude, é previsível que venha a constituir uma das principais causas de morbilidade e incapacidade total ou parcial durante $o$ século XXI. Em 2015 estima-se a existência de 415 milhões de pessoas com diabetes no mundo e, em 2040, estima-se que este valor ascenda a 642 milhões. Em 2015, a diabetes provocou 5 milhões de mortes a nível mundial. ${ }^{1}$

A prevalência da diabetes está a aumentar e em Portugal, segundo o Relatório Anual do Observatório Nacional da Diabetes, no ano de 2014, na população dos 20 aos 79 anos, a prevalência da diabetes estimada era de $13,3 \%$,

1. USF Coimbra Centro, Faculdade de Medicina da Universidade de Coimbra 2. USF Topázio, Faculdade de Medicina da Universidade de Coimbra. tendo crescido 1,4 pontos percentuais desde 2009. Em $2014,4 \%$ das mortes tiveram como origem esta doença e, em 2013, a diabetes representou cerca de oito anos de vida perdida por cada óbito por diabetes na população com idade $<70$ anos. $^{2}$

A intervenção mais estudada para o controlo da diabetes tipo 2 é a farmacológica, mas verifica-se que ao longo do tempo esta vai perdendo eficácia. ${ }^{3}$ Sabe-se também que a adesão à terapêutica nesta população nem sempre é a

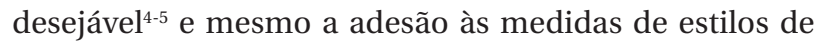
vida também está longe do que é recomendável. ${ }^{6}$ Assim, é importante o papel da educação aos diabéticos e a forma de o fazer tem sido estudada, ainda que em menos escala do que as intervenções farmacológicas.

Apesar de ser consensual que o conhecimento relacionado com aspetos de saúde não é uma condição suficiente 
para a mudança comportamental, este tipo de conhecimento é entendido como condição necessária. ${ }^{7}$ Folhetos informativos para as pessoas com diabetes têm sido considerados como a fonte mais utilizada de informação em saúde. ${ }^{8}$ Para estes serem compreensíveis, relevantes e informativos ${ }^{9}$ considera-se importante apresentarem uma boa inteligibilidade, com linguagem simples e uma organização e apresentação estruturadas, ${ }^{10}$ além de boa qualidade de conteúdos (serem baseados na evidência, isentos, revistos pelos pares e datados $)^{11}$ e ainda serem adequados à literacia de quem os lê. ${ }^{8}$

Assim, tendo em conta a importância de a informação escrita dada a pessoas com diabetes ser compreensível, o objetivo deste artigo é descrever o processo de validação de três folhetos para pessoas com diabetes.

\section{MÉTODOS}

A elaboração dos folhetos seguiu um processo sistemático de seleção de mensagens e desenvolvimento de conteúdos, revisão e avaliação.

Num primeiro momento foram revistos os folhetos disponíveis da área em Portugal ${ }^{12}$ e no estrangeiro. ${ }^{13-17}$ Foram produzidos três folhetos sobre três temas distintos - «O que é a diabetes», «Terapêutica da diabetes» e «Exercício físico e diabetes» - que seguiram as regras formais de institutos reconhecidos, nomeadamente a definição dos conteúdos de acordo com o público-alvo, uso de linguagem simples, clara, em frases pequenas e numa ordem lógica e apresentação espaçada e legível. ${ }^{10,18-21}$

Os materiais foram revistos por uma equipa multidisciplinar que incluiu médicos (endocrinologistas, de medicina interna e de medicina geral e familiar), nutricionistas, especialistas em exercício físico, enfermeiros, psicólogos, jornalistas médicos e pessoas com diabetes. A produção e a revisão consideraram o conteúdo e também a forma. Para esta última foram enviadas as diretrizes do National Health Service, Grã-Bretanha, ${ }^{21}$ para orientação.

Todas as sugestões feitas pelos peritos foram incorporadas, sendo eliminadas as frases que suscitavam mais controvérsia e dúvidas. Os folhetos foram seguidamente sujeitos a revisão de Português por peritos linguísticos.

Com o objetivo de validar a adequação cultural, a clareza, a acessibilidade e a compreensão das mensagens presentes nos materiais produzidos realizou se uma etapa de avaliação dos folhetos por pessoas com diabetes selecionadas por conveniência, com sorteio de um dia de consulta de diabetes na USF Rio Dão, em Santa Comba Dão (ACeS Dão Lafões, na ARS Centro), em que todas as pessoas com diabetes que tiveram consulta no dia 13 de outubro de 2014 e que consentiram participar no estudo fo- ram inquiridas. Após consentimento informado, os folhetos foram lidos pelos próprios e depois, com o investigador, foi questionado o que era percebido em cada frase e foram assinaladas as frases que suscitaram dúvidas. Foi usada uma grelha em que, para cada frase do folheto, era perguntado pelo investigador sucessivamente: «O que quer dizer para si esta frase?», «Tem alguma dúvida em relação a esta frase?» e «Tem alguma sugestão em relação a esta frase para a tornar mais clara?». Foram retiradas as frases que suscitavam dúvidas de interpretação e corrigidos erros menores segundo as sugestões obtidas.

Após este processo, para perceber melhor a evolução dos folhetos em termos de complexidade de frases escritas, foi feita a avaliação de inteligibilidade dos mesmos no início e depois do processo usando a ferramenta online gratuita LX-CEFR ${ }^{22}$ e procedeu-se a posterior cálculo da inteligibilidade segundo a fórmula de Flesch adaptada para Português. ${ }^{23}$ Utilizaram-se os cut-offs da fórmula original, sendo que os valores variam entre 100-75 (muito fácil), 7550 (fácil), 50-25 (difícil) e 25-0 (muito difícil), que correspondem, respectivamente, ao $1^{\circ}$ ciclo ( $1^{\circ}-4^{\circ}$ ano), $2^{\circ}$ e $3^{\circ}$ ciclos $\left(5^{\circ}-9^{\circ}\right.$ ano $)$, secundário $\left(10^{\circ}-12^{\circ}\right.$ ano $)$ e ensino superior.

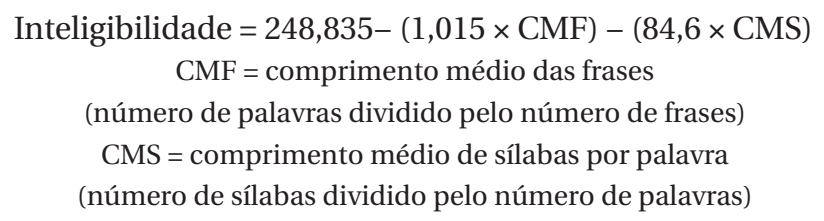

\section{RESULTADOS}

Um resumo das alterações decorridas ao longo do processo foi descrito na Figura 1.

$\mathrm{Na}$ fase de revisão de folhetos por peritos, que decorreu de 13-26 de setembro de 2014, foram contactados profissionais de diversas áreas com experiência em lidar com pessoas com diabetes e alguns com investigação na área, tendo sido recebidas 14 contribuições (cinco médicos de família, dois enfermeiros, dois médicos de medicina interna, um endocrinologista, um nutricionista, um perito em exercício físico, um psicólogo e um jornalista da área da saúde), bem como o contributo de uma pessoa com diabetes com formação superior.

Foram feitas todas as extensas alterações propostas e retiradas as frases que suscitavam dúvidas ou opiniões contrárias. Seguidamente foi feita uma revisão por dois peritos em língua portuguesa e realizadas algumas alterações na sintaxe e semântica.

Na fase de avaliação dos folhetos foi recebida a colaboração de pessoas da USF Rio Dão, que abrangia nesse ano 


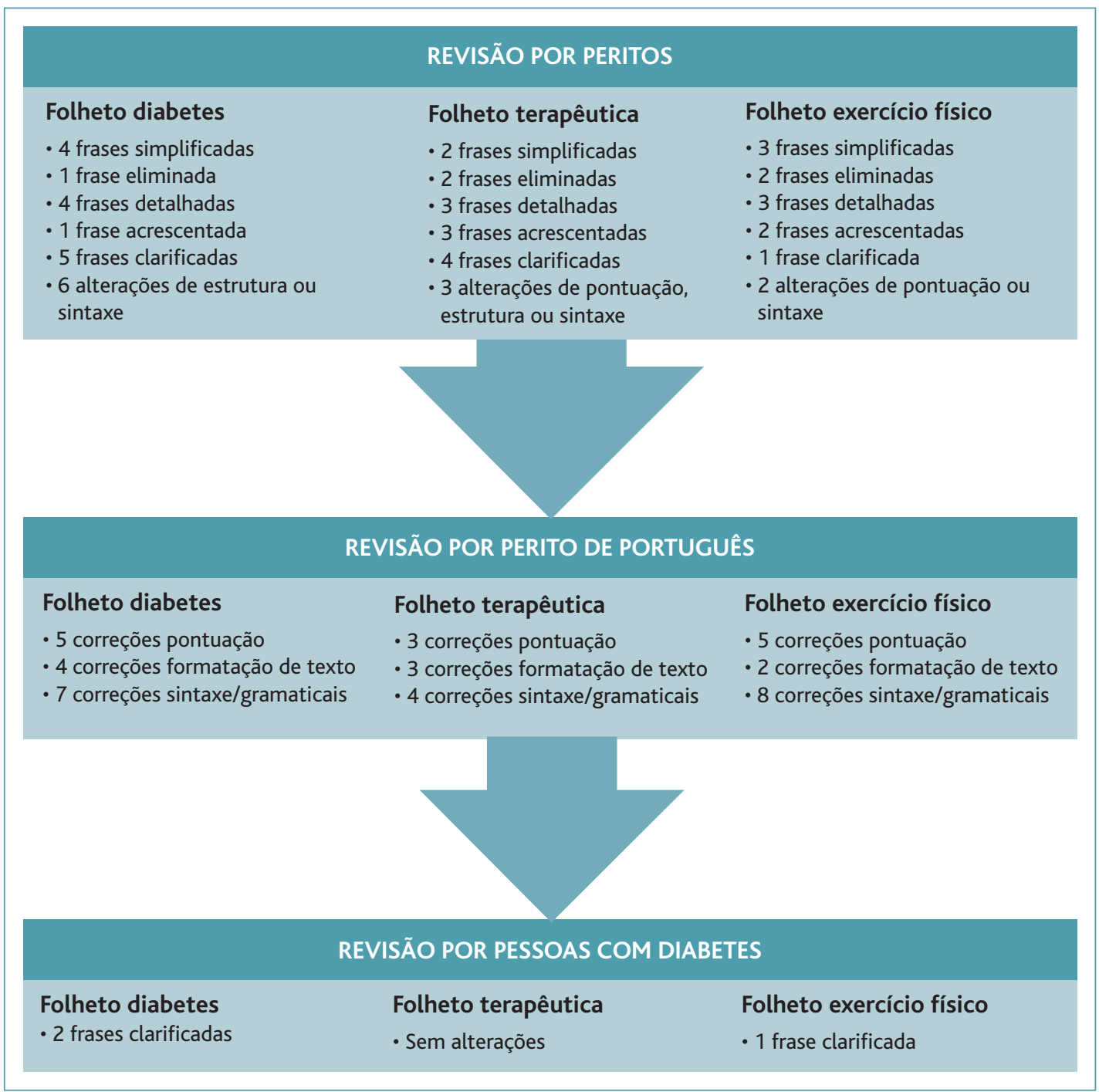

Figura 1. Fluxograma com as principais alterações efetuadas aos folhetos ao longo do processo de validação.

ca) e inferior para o folheto 3 (exercício físico), tendo diminuído em todos os folhetos desde o início da validação até ao final - no caso do folheto 1 à custa do número de sílabas/palavra e nos folhetos $2 \mathrm{e}$ 3 à custa do aumento do número de palavras/frase. O número de palavras e sílabas totais aumentaram em todos os folhetos desde a fase inicial até à final.

\section{DISCUSSÃO}

Neste estudo foram desenvolvidos três folhetos sobre diferentes aspetos da diabetes, que passaram por diversas fases de

uma população de 10.293 utentes (52,7\% mulheres), dos quais 1.047 com diagnóstico de diabetes tipo 2 (T90), sendo $50,24 \%$ homens com idade média de 66,38 anos. A amostra foi composta por 10 pessoas com diabetes, $50 \%$ do sexo masculino, com idade média 63,0 0 10,1 anos e uma formação média de 5,6 $\pm 4,5$ anos (Figura 2). Pediu-se que explicassem a sua compreensão das frases dos folhetos uma a uma, assinalando dúvidas e sugestões. Apenas dois reportaram dúvidas e sugestões, sendo clarificadas as três frases em questão.

Fez-se depois a avaliação da inteligibilidade dos folhetos, obtendo-se os resultados descritos no Quadro I para cada um dos folhetos no início e no final do processo, sendo o Índice de Flesch superior para o folheto 2 (terapêuti- validação. Após elaboração do esqueleto inicial foram validados por um grupo diversificado de peritos (oito profissões diferentes) e por peritos de língua. Depois foram avaliados por um grupo de pessoas com diabetes com igual distribuição entre os géneros (apesar de haver maior prevalência de homens com diabetes tipo $2,{ }^{2}$ as mulheres são mais frequentadoras dos cuidados de saúde primários), idade média de 63,0ะ10,1 anos, aproximada à média nacional de pessoas com diabetes ${ }^{2}$ e com formação muito variada (0-15 anos de formação). Esta validação foi essencialmente qualitativa por entrevista individual; daí ter-se escolhido a amostra num dia sorteado e serem feitas entrevistas até garantir uma amostra com formação académica suficientemente variada. Este processo não foi repe- 
tido depois das alterações pelo facto das mesmas terem sido insignificantes na forma e na extensão.

Estes folhetos sofreram muitas alterações durante a validação pelos peritos, como se pode verificar pelo aumento do número de palavras e sílabas totais e pelo grau de dificuldade de leitura (diminuição do índice de Flesch de inteligibilidade) em todos eles, provavelmente por se ter seguido a regra recomendada pelo $\mathrm{NHS}^{21}$ de considerar obrigatoriamente todas as contribuições de todos os peritos e por estes quererem torná-los mais corretos cientificamente, acrescentando descrições mais detalhadas.

Já na fase de avaliação pelas pessoas com diabetes sofreram poucas alterações, tendo a maioria referido compreender o que estava escrito. Admite-se um viés na resposta destas pessoas, as quais poderiam sentir-se condicionadas por serem questionadas por um médico com quem podiam não se sentir à vontade para admitir a não compreensão dos conceitos ou criticar a forma como estavam escritos. Outra hipótese teria sido elaborar um questionário objetivo sobre os conteúdos de cada frase, validar esse questionário e aplicá-lo de forma anónima e com autopreenchimento no sentido de perceber o que era efetivamente compreendido em cada frase, ou seja, por métodos quantitativos. Além disso, poder-se-ia ter avaliado se cada uma das frases trazia informação nova ou não. Mas este método aumenta a complexidade da avaliação por pessoas potencialmente com baixa literacia e, ainda assim, não garante totalmente a compreensão dos conteúdos, pelo que não foi utilizado. Além disso, teria sido importante envolver as pessoas com diabetes na validação dos folhetos desde a sua conceção, percebendo quais são as áreas que mais as preocupam e que mais valorizam, já que tem sido encontrado algum desfasamento em estudos entre os conteúdos dos folhetos e o que as pessoas gostariam efetivamente de saber. ${ }^{24-25}$ Em Portugal verificouse, em estudos anteriores, que as áreas da

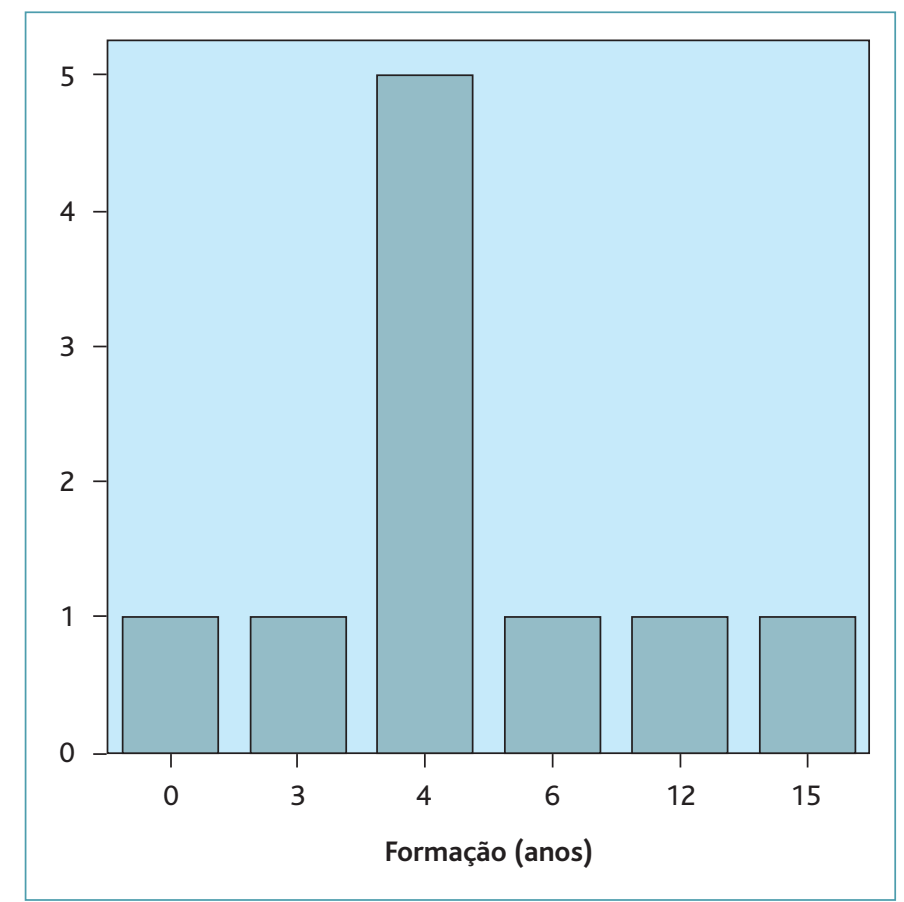

Figura 2. Distribuição dos anos de formação das pessoas com diabetes que aceitaram participar na fase de avaliação dos folhetos por número de anos de formação $(n=10)$.

identidade e as causas da diabetes parecem ser aquelas em que se regista menos conhecimento e que suscitam mais dúvidas nas pessoas com esta doença. Outros estudos re-

\section{QUADRO I. Resultado da avaliação de parâmetros de inteligibilidade dos três folhetos e resultado da fórmula de Flesch adaptada para o Português, ${ }^{23}$ antes e depois da validação,} obtidos através da ferramenta online LX-CEFR ${ }^{22}$

\begin{tabular}{|l|r|r|r|r|r|r}
\multirow{2}{*}{ Parâmetros } & \multicolumn{2}{|c|}{$\begin{array}{c}\text { Folheto } 1 \\
\text { Diabetes }\end{array}$} & \multicolumn{2}{c|}{$\begin{array}{c}\text { Folheto 2 } \\
\text { Terapêutica }\end{array}$} & \multicolumn{2}{c}{$\begin{array}{c}\text { Folheto 3 } \\
\text { Exercício físico }\end{array}$} \\
\cline { 2 - 7 } & Antes & Depois & Antes & Depois & Antes & Depois \\
\hline Número sílabas totais & 629 & 736 & 422 & 617 & 642 & 760 \\
\hline Número palavras totais & 303 & 344 & 197 & 290 & 286 & 343 \\
\hline Número frases totais & 17 & 20 & 18 & 18 & 17 & 18 \\
\hline Número letras/palavra & 4,71 & 4,87 & 4,95 & 4,93 & 5,13 & 5,12 \\
\hline Número sílabas/palavra (CMS) & 2,08 & 2,14 & 2,14 & 2,13 & 2,24 & 2,22 \\
\hline Número palavras/frase (CMF) & 17,82 & 17,2 & 10,94 & 16,11 & 16,82 & 19,06 \\
\hline Inteligibilidade (Flesch adaptado) & 54,77 & 50,33 & 56,69 & 52,29 & 42,26 & 41,68 \\
\hline Inteligibilidade (ano de escolaridade & $9^{\circ}$ ano & $9^{\circ}$ ano & $9^{\circ}$ ano & $9^{\circ}$ ano & $12^{\circ}$ ano & $12^{\circ}$ ano \\
correspondente) & & & & & &
\end{tabular}


fletiram o mesmo nas áreas do seu controlo e consequên$\operatorname{cias}^{26-29}$ e ainda dificuldades específicas em relação à operacionalização do exercício físico, ${ }^{30}$ que são focadas nos folhetos que elaborámos e por esta razão baseámo-nos nesses estudos e não fizemos a complexa avaliação das principais necessidades percebidas pelas pessoas com diabetes em todo o país.

As características da amostra de pessoas com diabetes tipo 2 serão de uma população com níveis de instrução relativamente baixos (cinco a seis anos de estudos, em média). Em trabalhos futuros será pertinente saber também a duração da doença em anos, pois esta poderá estar associada à possível tolerância à presença de termos técnicos nos folhetos e explicados habitualmente nas consultas pelos profissionais de saúde.

Alguns estudos, em contextos geográficos diferentes, utilizaram questionários construídos para fazer a avaliação da compreensão de folhetos, ${ }^{31}$ o que seria uma alternativa e poderia minimizar este viés e esta subjetividade. Ainda assim, o envolvimento de pacientes na validação dos folhetos é recomendado ${ }^{21} \mathrm{e}$ incentivado, ${ }^{9}$ sendo, por isso, foi um ponto forte desta validação.

$\mathrm{Na}$ avaliação posterior da inteligibilidade dos folhetos, os folhetos finais 1 e 2 apresentaram uma inteligibilidade ao nível do $9^{\circ}$ ano de escolaridade e o folheto 3 ao nível do $12^{\circ}$ ano de escolaridade, segundo a fórmula de Flesch adaptada para a língua Portuguesa.

Esta fórmula faz a correção do número de sílabas por palavra de Inglês para Português do Brasil, mas não tem em conta as diferenças linguísticas nem as diferenças dos sistemas de ensino na classificação por anos de escolaridade. Porém, parece haver correlação com outras escalas e os resultados são significativos para o Português do Brasil..$^{32}$ De qualquer forma, nunca foi validada para Português de Portugal pelo que deverá haver algum cuidado na sua interpretação literal, ${ }^{23}$ mas não existe outra forma melhor de medir a inteligibilidade em Português, ${ }^{33}$ sendo uma alternativa à tradução dos folhetos para Inglês e posterior análise. ${ }^{34}$

Existem também várias críticas a estas fórmulas por serem baseadas apenas na métrica. Por um lado, na área da saúde, a terminologia tem particularidades com uso de palavras mais longas e, por outro lado, com estas fórmulas não é avaliada a forma como a informação está disposta nas frases, ${ }^{9}$ na medida em que estes folhetos em particular têm várias frases compostas, com várias linhas enumeradoras separadas por parágrafos. Além disso, estas avaliações são focadas apenas nos folhetos e não têm em conta as pessoas que os vão ler, o seu conhecimento prévio e a sua interação com o folheto, não devendo, por isso, ser usadas isoladamente. ${ }^{9}$
Neste caso específico teria sido interessante usar também medidas estruturadas de avaliação da apresentação dos folhetos, como a ferramenta SAM ${ }^{35}$ que tem em conta os objetivos, o grafismo e os fatores culturais na sua avaliação. Não foi aplicada por não acrescentar muito aos esforços já feitos em termos de validação de conteúdos (por terem sido usados os princípios já enumerados na sua construção) e de legibilidade (que é baseada na fórmula de Flesch apresentada), sendo que acrescentaria apenas validação na parte gráfica, o que no caso destes folhetos não fazia tanto sentido por não usarem imagens nem gráficos. Poderia ter sido avaliada também a qualidade dos conteúdos, para o que estão a ser desenvolvidos instrumentos validados, mas estes ainda são muito recentes e centrados nos investigadores, pelo que se não aplicaram. ${ }^{9}$

Em termos de avaliação da literacia em saúde do público-alvo, em Portugal já estão validados instrumentos para a avaliar, ${ }^{36-38}$ o que pode ser interessante estudar e considerar em validações futuras, mas aqui não puderam ser utilizados por serem posteriores ao nosso estudo. Como nem sempre a simplificação de folhetos é suficiente para melhorar a compreensão, ${ }^{39}$ seria desejável desenvolver procedimentos de avaliação da inteligibilidade dos folhetos que controlassem simultaneamente o efeito das variações da literacia em saúde e avaliassem a aquisição dos conhecimentos adquiridos por meio da leitura dos mesmos, de forma a perceber mais diretamente o que nos interessa: se os folhetos se traduzem em conhecimentos adquiridos e funcionantes. ${ }^{40}$

Estes folhetos, segundo a avaliação da inteligibilidade feita, e com a ressalva das limitações já descritas, classificaram-se ao nível do $9^{\circ}-12^{\circ}$ ano. Nos EUA é recomendado que os folhetos tenham uma legibilidade entre o $6^{\circ}$ e $9^{\circ}$ ano, ${ }^{41}$ de acordo com a literacia nacional que, ainda assim, tem demonstrado ser superior à portuguesa. ${ }^{42-43}$ Tem sido demonstrado em estudos internacionais ${ }^{31,33}$ e em Portugal $^{34}$ que a inteligibilidade dos folhetos da área da saúde é inferior ao desejável, ou seja, são feitos por técnicos que afloram a informação na sua ótica, esquecendo a adaptação à literacia e à capacidade de compreensão populacional média a quem a informação se dirige. Nesta validação tentou-se adaptar os folhetos à compreensão do público-alvo pelo estudo-piloto realizado e que, mesmo assim, pode ser insuficiente.

Não existe uma cultura de validação e simplificação de folhetos em Portugal, havendo poucos estudos publicados de validação, ${ }^{44}$ avaliação ${ }^{34,40,45}$ e simplificação de materiais escritos. ${ }^{46}$ Este tipo de procedimento deve ser valorizado para melhorar a efetividade da informação escrita que é construída para a nossa população. Aponta-se, então, a 
necessidade do desenvolvimento de ferramentas realizadas no contexto Português para avaliação de inteligibilidade, qualidade de conteúdos, adaptação à literacia em saúde dos pacientes-alvo e, mais ainda, do nível de compreensão e melhoria da capacitação dos mesmos.

\section{CONCLUSÕES}

Foram elaborados três folhetos informativos para diabéticos tipo 2 seguindo um processo de desenvolvimento de conteúdos, segundo as recomendações existentes, revisão por peritos científicos e de língua e avaliação por pessoas com diabetes. Foi feita avaliação posterior por aplicação de fórmula de inteligibilidade que revelou um grau de legibilidade dos mesmos ao nível do $9^{\circ}-12^{\circ}$ ano.

\section{AGRADECIMENTOS}

Agradecemos a todos os peritos que gentilmente acederam em contribuir para a validação destes folhetos: João Filipe Raposo, Daniela Marado, Álvaro Coelho, Rosa Gallego, Daniel Pinto, Dilermando Sobral, Lina Costa, Maria Lurdes Tavares-Bello, Joana Moutinho, Adriana Baptista, Joana Grácio, Ana Filipa Cardoso, Joana Godinho, Jaime Carvalho e Silva, Tiago Villanueva.

Agradecemos às pessoas com diabetes e profissionais da USF Rio Dão, que participaram nesta validação.

\section{NOTA}

O texto integral destes Folhetos está disponível na versão digital da RPMGF onde poderá ser consultado.

\section{REFERÊNCIAS BIBLIOGRÁFICAS}

1. International Diabetes Federation. IDF diabetes atlas. 7th ed. IDF; 2015. ISBN 9782930229812

2. Observatório Nacional da Diabetes. Diabetes: factos e números 2014 - relatório anual. Lisboa: Sociedade Portuguesa de Diabetologia; 2015.

3. Holman RR, Paul SK, Bethel MA, Matthews DR, Neil HA. 10-year follow-up of intensive glucose control in type 2 diabetes. N Engl J Med. 2008;359(15):1577-89.

4. Morris AD, Boyle DI, McMahon AD, Greene SA, MacDonald TM, Newton RW. Adherence to insulin treatment, glycaemic control, and ketoacidosis in insulin-dependent diabetes mellitus. Lancet. 1997;350(9090):1505-10.

5. Donnan PT, MacDonald TM, Morris AD. Adherence to prescribed oral hypoglycaemic medication in a population of patients with type 2 diabetes: a retrospective cohort study. Diabet Med. 2002;19(4):279-84.

6. Toobert DJ, Hampson SE, Glasgow RE. The summary of diabetes self-care activities measure: results from 7 studies and a revised scale. Diabetes Care. 2000;23(7):943-50.

7. Ogden J. Health psychology: a textbook. London: Open University Press; 2012. ISBN 9780335243839

8. Kenny T, Wilson RG, Purves IN, Clark J, Newton LD, Newton DP, et al. A PIL for every ill? Patient information leaflets (PILs): a review of past, present and future use. Fam Pract. 1998;15(5):471-9.

9. Luk A, Aslani P. Tools used to evaluate written medicine and health information: document and user perspectives. Health Educ Behav. 2011;38(4):389-403.

10. School of Public Health. Assessing and developing health materials [Internet]. Boston: Harvard T. H. Chan [cited 2015 Dec 11]. Available from: http://www.hsph.harvard.edu/healthliteracy/practice/innovative-actions/

11. Meredith $P$, Emberton $M$, Wood $C$. New directions in information for patients. BMJ. 1995;311(6996):4-5.

12. Associação Protetora dos Diabéticos de Portugal. A pessoa com diabetes
[Internet]. Lisboa: APDP [cited 2016 Mar 6]. Available from: http://www.apdp.pt/diabetes/a-pessoa-com-diabetes

13. Tidy C. Diabetes mellitus. Patient.info [homepage]; 2016 [cited 2016 Mar 6]. Available from: http://patient.info/doctor/diabetes-mellitus

14. NHS Borders Diabetes Network. Patient information: leaflets regarding diabetes. NHS BDN [homepage] [cited 2016 Mar 6]. Available from: http://www.bordersdiabetesnetwork.scot.nhs.uk/patient-information

15. Diabetes in General Practice. Diabetes resource pack: information, guidelines and leaflets. DiGP [homepage]; 2012 [cited 2016 Mar 6]. Available from: http://www.digp.ie/resource/

16. American Family Physician. Diabetes: type 2 [Internet]. Washington: American Academy of Family Physicians; 2017 [cited 2016 Mar 6]. Available from: http://www.aafp.org/afp/topicModules/viewTopicModule.htm?topicModuleld=7\#5

17. Easyhealth. Diabetes leaflets. London: Easyhealth [homepage]; 2010 [cited 2016 Mar 6].Available from: http://www.easyhealth.org.uk/listing/diabetes-(leaflets)

18. National Institutes of Health. Clear and simple: developing effective print materials for low literate readers. Washington: U.S. Department of Health and Human Services; 1994.

19. Health Sciences Libraries. Creating patient education materials [Internet]. Minneapolis: University of Minnesota; 2009 [cited 2015 Dec 11]. Available from: https://hsl.lib.umn.edu/biomed/help/creating-patient-education-materials

20. Centers for Medicare \& Medicaid Services. Toolkit for making written material clear and effective [Internet]. Baltimore: CMMS; 2012 [cited 2015 Dec 11].Available from: https://www.cms.gov/Outreach-and-Education/Outreach/WrittenMaterialsToolkit/index.html?redirect=/WrittenMaterialsToolkit

21. NHS Brand Guidelines. Tools and resources: patient information [Internet]. London: NHS England; 2016 [cited 2016 Mar 6]. Available from: http://www.nhsidentity.nhs.uk/tools-and-resources/patient-information

22. Departamento de Informática. LX - CEFR [homepage]. Universidade de Lisboa [cited 2016 Mar 10]. Available from: http://nlx-server.di.fc.ul.pt/ jrodrigues/camoes/indexLXCENTER.html

23. Scarton CE, Aluísio SM. Análise da inteligibilidade de textos via ferramentas de processamento de língua natural: adaptando as métricas do Coh-Metrix para o Português. Linguamática. 2010;2(1):45-61.

24. Fitzmaurice DA, Adams JL. A systematic review of patient information leaflets for hypertension. J Hum Hypertens. 2000;14(4):259-62.

25. Reid JC, Klachko DM, Kardash CA, Robinson RD, Scholes R, Howard D. Why people don't learn from diabetes literature: influence of text and reader characteristics. Patient Educ Couns. 1995;25(1):31-8.

26. Sousa MR, Mclntyre T. Conhecimento do diabético sobre a doença e a repercussão no tratamento [Knowledge of the diabetic patient about the disease and its impact on treatment]. Rev Bras Promoção Saúde. 2012;21(4):281-9. Portuguese

27. Neves MS. Adesão ao regime terapêutico em pessoas com diabetes mellitus tipo 2: importância dos conhecimentos e da motivação [Dissertation]. Coimbra: Escola Superior de Enfermagem de Coimbra; 2014.

28. Sousa MR, Mclntyre T, Martins T, Silva E. Questionário dos Conhecimentos da Diabetes (QCD): propriedades psicométricas [Diabetes Knowledge Questionnaire (DKQ): psychometric properties]. Rev Port Saúde Pública. 2015;33(1):33-41. Portuguese

29. Baptista LM. Representações cognitivas da diabetes, conhecimentos acerca da doença e autocuidados: um estudo com diabéticos tipo II controlados e não controlados [Dissertation]. Lisboa: Universidade Católica Portuguesa; 2014.

30. Laranjo L, Neves AL, Costa A, Ribeiro RT, Couto L, Sá AB. Facilitators, barriers and expectations in the self-management of type 2 diabetes: a qualitative study from Portugal. Eur J Gen Pract. 2015;21(2):103-10.

31. Tong $V$, Raynor DK, Aslani P. Design and comprehensibility of over-thecounter product labels and leaflets: a narrative review. Int J Clin Pharm. 2014;36(5):865-72. 
32. Martins TB, Ghiraldelo CM, Nunes MG, Oliveira Jr ON. Readability formulas applied to textbooks in Brazilian Portuguese. São Carlos: Instituto de Ciências Matemáticas de São Carlos-USP; 1996.

33. Pires $C$, Vigário $M$, Cavaco A. Readability of medicinal package leaflets: a systematic review. Rev Saude Publica. 2015;49:4.

34. Cavaco AM, Várzea D. Contribuição para o estudo da leitura de folhetos informativos nas farmácias Portuguesas [Contribution to the study of information leaflets readability in Portuguese pharmacies]. Rev Port Saúde Pública. 2010;28(2):179-86. Portuguese

35. Doak CC, Doak LG, Root JH. Teaching patients with low literacy skills. Am J Nurs. 2012;96(12):16M.

36. Paiva D, Santos O, Silva S, Severo M, Ferreira P, Azevedo A, et al. Validação de um instrumento de avaliação de literacia em saúde e prevalência de literacia em saúde adequada na população portuguesa. Portugal Saúde em Números. 2014;(3):36.

37. Pedro AR, Amaral $O$, Escoval A. Literacia em saúde, dos dados à ação: tradução, validação e aplicação do European Health Literacy Survey em Portugal [Health Literacy, from data to action: Translation, validation and application of the European Health Literacy Survey in Portugal]. Rev Port Saúde Pública. 2016;34(3):259-75. Portuguese

38. Paiva D, Silva S, Severo M, Ferreira P, Santos O, Lunet N, et al. Cross-cultural adaptation and validation of the health literacy assessment tool METER in the Portuguese adult population. Patient Educ Couns. 2014;97(2):269-75.

39. Nielsen-Bohlman L, Panzer AM, Kindig DA, editors. Health literacy: a prescription to end confusion. Washington:The National Academies Press; 2004. ISBN 0309529263

40. Cavaco A, Santos AL. Evaluation of health literacy and the readability of information leaflets. Rev Saúde Pública. 2012;46(5):918-22.

41. Food and Drug Administration, Center for Drug Evaluation and Research, Center for Biologics Evaluation and Research. Guidance: useful written consumer medication information (CMI). Rockville: U.S. Department of Health and Human Services; 2006.
42. Benavente A, Rosa A, Costa AF, Ávila P. A literacia em Portugal: resultados de uma pesquisa extensiva e monográfica. Lisboa: Fundação Calouste Gulbenkian, Conselho Nacional de Educação, 1996. ISBN 9723107139

43. Gomes MC, Ávila P, Sebastião J, Costa AF. Novas análises dos níveis de literacia em Portugal: comparações diacrónicas e internacionais. In: Sociedade Portuguesa: passados recentes, futuros próximos (actas do $4^{\circ} \mathrm{Con}$ gresso Português de Sociologia). Coimbra: Associação Portuguesa de Sociologia; 2002.

44. Goes AR, Câmara G, Loureiro I, Bragança G, Nunes LS, Bourbon M. «Papa Bem»: investir na literacia em saúde para a prevenção da obesidade infantil [«Papa Bem»: investing in health literacy for childhood obesity prevention]. Rev Port Saúde Pública 2015;33(1):12-23. Portuguese

45. Pires C, Cavaco A, Martins F, Vigário M. Using an automatic tool to identify potential readability issues in a large sample of medicinal package inserts. Methods Inf Med. 2015;54(4):379-81.

46. Cavaco A, Pires C. Improving package leaflet information: potential users and physicians opinions [abstract]. Res SocAdm Pharm. 2012;8(6):e50-e51.

\section{COMISSÃO DE ÉTICA}

Estudo realizado com pareceres favoráveis da Comissão de Ética da Faculdade de Medicina da Universidade de Coimbra e da ARS Centro.

\section{CONFLITO DE INTERESSES}

O autor declara não possuir qualquer tipo de conflito de interesses.

\author{
ENDEREÇO PARA CORRESPONDÊNCIA \\ Inês Rosendo \\ USF Coimbra Centro \\ inesrcs@gmail.com
}

Recebido em 22-08-2016

Aceite para publicação em 23-07-2017

\section{ABSTRACT}

\section{VALIDATION OF THREE PATIENT INFORMATION LEAFLETS ABOUT DIABETES AND EXERCISE}

Aims: Development and evaluation of three patient information leaflets for people with type 2 diabetes

Methods: The leaflets were designed in accordance with existing recommendations for the development of patient education materials. This included review of the material by scientific and linguistic experts and by people with diabetes. The Flesch readability formula adapted to Portuguese was applied.

Results: In the review phase, 14 scientific experts, one person with diabetes and two language experts provided comments on the material and suggested changes were made. In the evaluation phase, contributions were received from 10 people with diabetes, $50 \%$ of whom were male, with a mean age of $63.0 \pm 10.1$ years and a mean level of education of $5.6 \pm 4.5$ years. The evaluation of the readability suggested that the material was at the ninth to twelfth grade comprehension level. In all leaflets, the number of total words and syllables increased from the beginning to the end of the development process.

Conclusions: This study documents the development and evaluation of leaflets for patients with Type 2 diabetes in Portugal. It used scientific and language validation by experts who made major changes in the text. It involved patients in the validation and final evaluation of readability, which showed that the final reading level was probably too high. We suggest tools for the development of patient information leaflets in the Portuguese context to assess intelligibility, quality of content, and health literacy. Leaflets should be adapted to the needs of patients. An assessment of their level of understanding and enablement is required.

Keywords: Pamphlets; Patient education handout; Diabetes mellitus; Therapeutics; Exercise 


\section{ANEXO I}

\section{DIABETES - O QUE É?}

- Diabetes é uma doença em que o "açúcar" que existe no sangue (glicemia) está acima do normal.

Posso ter o diagnóstico de diabetes quando os valores da glicemia são:

- superiores a $126 \mathrm{mg} / \mathrm{dl}$, em jejum

ou

o superiores a $200 \mathrm{mg} / \mathrm{dl}$, em qualquer momento do dia.

\section{É IMPORTANTE SABER QUE:}

A insulina é uma substância que faz o organismo utilizar a glicose para a produção de energia. Sem a insulina, o corpo não conseguiria usar a glicose e morreria.

Quando o açúcar está em excesso no sangue (hiperglicemia), isso significa que o corpo não utilizou todo o açúcar que foi ingerido. Isto pode acontecer porque:

- o pâncreas produz menos insulina ou

- a insulina que produz não causa o efeito pretendido ou

- as duas causas acima associadas.

A falta de açúcar no sangue (hipoglicemia, ou seja, valores inferiores a $70 \mathrm{mg} / \mathrm{dl}$ ) e sintomas como cansaço, dor de cabeça, visão turva ou tonturas indicam que é preciso ingerir alimentos.

QUAIS AS CAUSAS DA DIABETES TIPO 2?

Os principais fatores de risco são:

- excessos e erros alimentares, com consumo exagerado de açúcar (que está em excesso em alguns alimentos, tais como cereais de pequeno-almoço, bolos, doces, chocolates, rebuçados, mel, compotas, marmelada, frutas em calda, chocolate para o leite, bolachas, pão de forma e sumos/refrigerantes),

- falta de atividade física (sedentarismo).

$\mathrm{O}$ que pode agravar e aumentar o risco de problemas relacionados com a diabetes?

- Estar gordo (ter excesso de peso ou obesidade), sobretudo na barriga;

- Ter excesso de gorduras no sangue (colesterol) e tensão arterial alta;

- Fumar;

- Não fazer atividade física.

\section{CONSEQUENNCIAS: O QUE PODE ACONTECER?}

- As artérias adoecem mais cedo, principalmente as do cérebro, coração, olhos e rins, levando ao aparecimento de doenças nestas partes do corpo.

- Os nervos, que conduzem sensações dolorosas, entre outras, são danificados. A diminuição da sensibilidade pode contribuir para o aparecimento de pequenas lesões em todo o corpo sem se aperceber, principalmente, feridas nos pés. 


\section{ANEXO II \\ DIABETES: COMO SE TRATA}

Para controlar os níveis de açúcar no sangue e prevenir complicações, é fundamental:

- Caminhar (ou realizar outra atividade física) todos os dias e perder peso (sobretudo volume na zona da barriga);

- Fazer mais de quatro refeições por dia e consumir menos açúcar (que está em excesso em alguns alimentos, tais como bolos, doces, compotas, chocolate, bolachas, pão de forma, cereais de pequeno-almoço e sumos/refrigerantes) e menos gorduras de origem animal (como manteiga e banha);

- Tomar sempre a medicação aconselhada pelo médico.

\section{MEDICAMENTOS UTILIZADOS:}

1. Antidiabéticos orais (comprimidos para a diabetes), que baixam o açúcar no sangue, pois:

a) diminuem a absorção de açúcar no intestino;

b) aumentam a produção de insulina pelo pâncreas;

c) ajudam o corpo a fazer melhor uso da insulina.

Nota: Existe um novo medicamento injetável que atua da mesma forma.

2. Insulina injetada, que substitui a que o seu pâncreas não produz.

\section{MEDICAMENTOS E DIABETES: INFORMAÇÕES IMPORTANTES}

Os antidiabéticos orais devem ser tomados à hora e na dose que o seu médico indicou.

Os medicamentos fazem efeito durante algumas horas. Se não os tomar todos os dias, deixam de fazer $o$ efeito desejado.

Quando os valores do açúcar no sangue se aproximam de valores normais, não deixe de tomar a medicação sem aconselhamento do seu médico.

Outros medicamentos podem alterar o efeito dos medicamentos para a diabetes, por isso, não deve tomar medicamentos sem o conselho do seu médico. No entanto, se já os está a tomar, não se esqueça de informar o seu médico.

Os medicamentos ajudam a controlar a diabetes, mas não fazem tudo sozinhos. A alimentação e o exercício também são importantes. 


\section{ANEXO III \\ DIABETES: A IMPORTÂNCIA DO EXERCÍCIO}

No tratamento da sua diabetes, para controlar os níveis de açúcar no sangue e prevenir complicações, é fundamental:

- Caminhar (ou realizar outra atividade física) todos os dias e perder peso (sobretudo o volume na zona da barriga);

- Fazer mais de quatro refeições por dia e consumir menos açúcar (que está em excesso em alguns alimentos, tais como bolos, doces, compotas, chocolate, bolachas, pão de forma, cereais de pequeno-almoço e sumos/refrigerantes) e menos gorduras de origem animal (como manteiga e banha);

- Tomar sempre a medicação aconselhada pelo médico.

\section{PORQUE DEVO FAZER EXERCÍCIO FÍSICO?}

O exercício físico vai estimular a produção de insulina que faz os músculos aumentarem a utilização de açúcar (glicose), diminuindo os níveis de açúcar no sangue.

Apesar de não ser sempre necessário, pode fazer o controlo do açúcar no sangue antes e depois da prática de exercício para melhor perceber os seus benefícios.

A prática de exercício tem outros benefícios:

- melhora a saúde do coração, das artérias e veias (cardiovascular) e ativa a circulação;

- fortalece os músculos, ajuda na perda de peso;

- melhora o humor, a qualidade do sono e a autoconfiança;

- diminui a tensão arterial.

\section{COMO DEVO PRATICAR EXERCÍCIO FÍSICO?}

Não precisa de se tornar um atleta! Pode optar por vários tipos de exercícios, consoante a sua idade, gosto e condição física.

Sempre que pretender praticar exercício físico, especialmente se não está habituado, deve consultar o seu médico.

Pode começar por caminhadas diárias de duração crescente até chegar a 40 minutos ou mesmo a 1 hora por dia. Se preferir exercícios de ginásio, informe os profissionais de que tem diabetes.

É importante usar calçado confortável e meias adequadas quando praticar exercício, bem como fazer um pequeno aquecimento com alongamentos antes de começar.

Pergunte ao seu médico se deve vigiar a sua glicemia (nível de açúcar no sangue) antes e após a prática de exercício físico. É importante que não pratique exercício físico em jejum. 